Pacific Journal of Mathematics

aPOLOGIES FOR FUNCTION SPACES 


\title{
TOPOLOGIES FOR FUNCTION SPACES
}

\author{
Richard Arens and James Dugundi
}

1. Introduction. Let $Z^{Y}$ denote the class of continuous functions (or "mappings," or "maps")

$$
f: Y \rightarrow Z
$$

of a topological space $Y$ into another $Z$. A great variety of topologies $t$ may be introduced into $Z^{Y}$ making it into a topological space $Z^{Y}(t)$. The topologies we deal with in this paper can be classified by using the notion of "continuous convergence" of directed sets (generalized sequences) $f_{\mu}$ in $Z^{Y}$ as follows: with no reference to any topology $Z Y$, we can say $f_{\mu}$ converges continuously (Frink [1]; Kuratowski [2]) to $f\left(f_{\mu}\right.$ and $f$ are elements of $\left.Z^{Y}\right)$ if

$$
f_{\mu}\left(y_{\nu}\right) \rightarrow f(y)
$$

whenever $y_{\nu} \rightarrow y$ in $Y$. (We use the " $\rightarrow$ " for convergence as in (1.2), as well as for indicating the domain-range relation as in (1.1). The context prevents confusion.) We can classify the topologies $t$ for $Z^{Y}$ according as to whether

$$
\text { convergence in } Z^{Y}(t) \text { implies continuous convergence }
$$

or

$$
\text { continuous convergence implies convergence in } Z^{Y}(t) \text {. }
$$

Certainly there are other topologies possible in $Z^{Y}$, but we do not discuss these. There may be a topology $t$ satisfying both (1.3) and (1.4), but if so it is unique; see (5.6).

An apparently different approach to the same classification is suggested by homotopy theory. Beside $Y$ and $Z$, consider a third space $X$. For a function $g$ defined on $X \times Y$ with values in $Z$, we can define $g^{*}(x)$ mapping $X$ into $Z^{Y}$ by setting $g^{*}(x)(y)=g(x, y)$. Then a topology $t$ for $Z^{Y}$ may be such that, for any $X$,

$$
\text { if } g \text { is continuous, then } g^{*} \text { is continuous, }
$$

or

$$
\text { if } g^{*} \text { is continuous, then } g \text { is continuous. }
$$

Received May 12, 1950.

Pacific J. Math. 1(1951), 5-31. 
It is proved ((2.4), (2.5)) that (1.5) is equivalent to (1.3) and (1.6) is equivalent to (1.4). We call the former class of topologies proper, and the latter admissible.

The following questions about this class of topologies in $Z^{Y}$ are considered in this paper: What are the relations (in the sense of the conventional partial ordering of topologies) of the proper topologies to the admissible topologies? What can be said about the order-type of the proper topologies? of the admissible topologies?

We write $s \leq t$ if $s$ and $t$ are topologies such that a set open in $Z^{Y}(s)$ is open in $Z^{Y}(t)$. Then (a) if $s \leq t$ and $t$ is proper, $s$ is proper; (b) if $s \leq t$ and $s$ is admissible, so also is $t$; (c) if $s$ is proper and $t$ is admissible, then $s \leq t$; (d) there is at most one proper admissible topology, and such a topology is both the greatest proper and least admissible topology.

The proper topologies form a principal ideal in the lattice of all topologies for $Z^{Y}$; thus there is always a greatest proper topology. The admissible topologies are much more disorganized. We state some findings for the special case in which $Z$ is the real line. (e) When $Y$ is not locally compact, but is completely regular, there is no least admissible topology and (hence) no proper admissible topology; (f) if $Y$ is a metric space, not locally compact, then there always exists a pair of admissible topologies none of whose common lower bounds are admissible.

When $Y$ is locally compact, there does exist a proper admissible topology, as is well known, which we call the $k$-topology (see below (4.3)). We ask:To what extent do any of these properties of the $k$-topology persist when $Y$ is not locally compact? It is always proper, but sometimes not the greatest of the proper topologies even if $Y$ is completely regular. Admissibility does not often persist (See (c), above).

We consider a special class of topologies, the set-open topologies, whose definition is patterned after that of the $k$-topology except that arbitrary families $\{A\}$ of sets are admitted. We determine fairly complete criteria as to whether a given one is proper or admissible. The $k$-topology is always the greatest proper setopen topology, when $Z$ is metric, and also the g.l.b. of all admissible set-open topologies.

A subclass of the set-open topologies are the $\sigma$-topologies defined in terms of coverings (just as the $k$-topology is definable in terms of the covering by open sets with compact closure when $Y$ is compact). These topologies are admissible, and for any pair there is a common lower bound.

Considering that the space $F$ of closed subsets of $Y$ can be regarded as a function space, we felt it appropriate to point out that the usual Hausdorff topology, even when $Y$ is a compactum, is not proper, and that $k$-topology is not a Hausdorff topology.

One interesting by-product of our investigation of admissible topologies is that it enables us to answer in the negative, surprisingly enough, the following simple 
question about topological products:

Let $Y$ be a space, let $s, t$ be two topologies for a set $X$, and let $u$ be the greatest lower bound of the topologies $s$ and $t$. Then is the (product) topology of

$$
X(u) \times Y
$$

the greatest lower bound of the topologies of $X(s) \times Y, X(t) \times Y$ ?

Finally, we determine a necessary and sufficient condition that, when $Y$ is a locally compact regular space, $X$ is a set, and a topology $t$ has been given to $X \times Y$, a topology $s$ can be found for $X$ such that $t$ is the product topology of

$$
X(s) \times Y \text {. }
$$

2. Admissible topologies and proper topologies. By a space $Y$ we shall mean a set $Y$ in which certain subsets, including $Y$ and the empty set, are designated as open, and which have the property that their finite intersections and arbitrary unions are also open; no separation axioms are assumed. A basis for a space $Y$ is a collection $\sigma$ of open sets such that any open set in $Y$ can be represented as the union of sets of $\sigma$; a subbas is for the space $Y$ is a collection of open sets which, together with their finite intersections, form a basis. Compactness in this paper shall always be the bicompactness of Alexandroff-Hopf [1]; a space with the property that every infinite subset has a limit point is called Fréchet compact. A space $Y$ is locally compact if every point lies in an open set having compact closure. $Y$ is completely regular if, given any $y \in Y$ and open $U$ with $y \in U$, there exists a continuous real-valued function $f$ satisfying $f(y)=1$ and $f(x)=0$ for $x \notin U$. If $Y_{1}$ and $Y_{2}$ are two spaces, the topological product $Y_{1} \times Y_{2}$ is the space whose points are all collections of ordered pairs $\left(y_{1}, y_{2}\right), y_{1} \in Y_{1}, y_{2} \in Y_{2}$, and in which a basis consists of all sets of form $\left(U_{1} \times U_{2}\right), U_{i}$ being open in $Y_{i}, i=1,2$ 。

If $Y$ and $Z$ are two spaces, the symbol " $f: Y \rightarrow Z$ "will always denote a continuous mapping of $Y$ into $Z$; the totality of all such continuous maps will be written $Z^{Y}$. Various topologies can be introduced into the set $Z^{Y}$; a set $Z^{Y}$ with a topology is called a function space. In this section, we shall single out two important types of topologies in $Z Y$, and give elementary consequences of the definitions.

(2.1) DEFINITION. Let $Z$ and $Y$ be two given spaces. A topology $t$ in $Z^{Y}$ is called admissible if the mapping $\omega(y, f)=f(y)$ of $Y \times Z^{Y}$ into $Z$ is continuous in $y$ and $f$.

The set $Z^{Y}$ with the topology $t$ will be denoted by $Z^{Y}(t)$, but when no ambiguity is involved regarding the topology $t$ under discussion, the $(t)$ will be omitted. The mapping $\omega$ will be called the evaluation mapping.

We now make the following observation. Let $X, Y, Z$, be three spaces and $g$ a 
mapping of $X \times Y$ into $Z$. Setting $g^{*}(x)(y)=g(x, y)=f_{x}(y)$ and varying $x$, we can evidently regard $g^{*}$ as a mapping of $X$ into $Z^{Y}$. Conversely, if we have a mapping $g^{*}$ of $X$ into $Z^{Y}$, we can write $g(x, y)=g^{*}(x)(y)=f_{x}(y)$ and regard $g$ as a mapping of $X \times Y$ into $Z$. Two maps $g$ and $g^{*}$ related as just described will be called associated.

We can now show the intimate relationship between mappings of cartesian products and admissible topologies in function spaces: the continuity of any $g^{*}$ implies the continuity of the associated $g$.

(2.2) THEOREM. Let $Z$ and $Y$ be two given spaces. A topology $t$ in $Z$ is admissible if and only if

(2.21) for every space $X, g^{*}: X \rightarrow Z^{Y}(t)$ implies $g: X \times Y \rightarrow Z$, where $g$ is the associated mapping.

Proof. Assume $t$ is admissible, and $g^{*}: X \rightarrow Z^{Y}(t)$. Define

$$
h: Y \times X \rightarrow Y \times Z^{Y}(t) \text { by } h(x, y)=\left(g^{*}(x), y\right) .
$$

If $\omega$ is the evaluation map, we have $\omega h: Y \times X \rightarrow Z$, and it is not hard to see that $\omega h$ is the mapping associated with $g^{*}$. Hence, (2.21) holds.

Assume now (2.21) holds. In particular, select $X=Z^{Y}(t)$ and the identity map $I^{*}: Z Y(t) \rightarrow Z^{Y}(t)$; by (2.21) this means the associated map of $Y \times Z^{Y}(t)$ into $Z$ is continuous, and this associated mapping is precisely the evaluation mapping.

The other important class of topologies in $Z^{Y}$ is given in the following definition.

(2.3) DEFinition. Let $Z$ and $Y$ be two spaces. A topology $t$ in $Z^{Y}$ is proper if for every space $X, g: X \times Y \rightarrow Z$ implies $g^{*}: X \rightarrow Z^{Y}(t)$, where $g^{*}$ is the associated map.

An extremely useful equivalent formulation of the notion "proper" can be given which is based on directed sets and continuous convergence. We therefore insert an explanatory paragraph (cf. Birkhoff [2]).

A directed system $\triangle$ is a partially ordered system with the property that for any $\mu, \mu^{\prime} \in \Delta$, there exists a $\mu^{\prime \prime} \in \Delta$ with $\mu^{\prime \prime} \geq \mu, \mu^{\prime \prime} \geq \mu^{\prime}$. Every directed system $\triangle$ gives rise to a directed space $\Delta^{\prime}$ by addition of one ideal point $\infty$ satisfying $\infty \geq \mu$ for all $\mu \in \Delta$. The topology in $\Delta^{\prime}=\Delta \cup\{\infty\}$ is obtained by defining all $\mu$ to be open sets, and neighborhoods of $\infty$ to be all sets of form $\left\{\mu ; \mu \geq \mu^{\prime}\right.$ for some $\left.\mu^{\prime}\right\}, \mu^{\prime} \in \Delta$. If $\Gamma=\{\nu\}$ is another directed system, the set

$$
\Delta \times \Gamma=(\mu, \nu)
$$

of all pairs is also a directed system if we define $(\mu, \nu) \geq\left(\mu^{\prime}, \nu^{\prime}\right)$ whenever both $\mu \geq \mu^{\prime}$ and $\nu \geq \nu^{\prime}$. A $(\Delta-)$ directed set in a space $Y$ is a function on a 
directed system $\Delta$ with values in $Y$, and is denoted by $\left\{y_{\mu}\right\}_{\mu \epsilon \Delta}$ or more briefly by $\left\{y_{\mu}\right\}$; a directed set $\left\{y_{\mu}\right\}$ converges to $y$ (in symbols, $y_{\mu} \rightarrow y$ ) if for every neighborhood $V$ of $y$ there exists a $\mu^{\prime} \in \Delta$ with $y_{\mu} \in V$ for all $\mu \geq \mu^{\prime}$. Furthermore, we have $g: Y \rightarrow Z$ if and only if for every directed set $\left\{y_{\mu}\right\}, y_{\mu} \rightarrow y$ implies $g\left(y_{\mu}\right) \rightarrow g(y)$ (see Tukey $\left.[1, \mathrm{p} .28]\right)$. Let $\left\{f_{\mu}\right)$ be a directed set in the set $Z^{Y}$; $f_{\mu}$ converges continuously to $f \in Z^{Y}$ if for every $y$ and every neighborhood $W$ of $f(y)$ there is a $\mu^{\prime}$ and a neighborhood $V$ of $y$ such that for $\mu \geq \mu^{\prime}$ we have $f(V) \subset W$. (This definition is equivalent to that made in Section 1.) Notice that the idea of continuous convergence does not require any topology in $Z^{Y}$.

With these preliminaries, we prove the following result.

(2.4) TheOREM. Let $Z$ and $Y$ be two spaces. A topology $t$ in $Z^{Y}$ is proper if and only if for every directed system $\triangle$ and every $\triangle$-directed set $\left\{f_{\mu}\right\}$ in $Z^{Y}(t)$, the continuous convergence of $f_{\mu}$ to $f$ implies $f_{\mu} \rightarrow f$ according to $t$.

Proof. Suppose, first, $t$ is proper and let $f_{\mu}$ converge continuously to $f,\left\{f_{\mu}\right\}$ being directed by $\Delta$. Let $\Delta^{\prime}$ be the corresponding directed space. Then define $g(\mu, y)=f_{\mu}(y), g(\infty, y)=f(y)$. Now we have $g: \Delta^{\prime} \times Y \rightarrow Z$, by the definition of continuous convergence. Hence $f_{\mu}=g^{*}(\mu) \rightarrow g^{*}(\infty)=f$ as desired.

Now suppose continuous convergence always implies convergence, and suppose we have $g: X \times Y \rightarrow Z$. Suppose $x_{\mu} \rightarrow x$ in $X$. It is easy to see that $g^{*}\left(x_{\mu}\right)$ converges continuously to $g^{*}(x)$ since $g$ is continuous. Thus $g^{*}\left(x_{\mu}\right) \rightarrow g^{*}(x)$ in $Z$. This proves that we have $g^{*}: X \rightarrow Z^{Y}$. Consequently $t$ is proper.

We remark that if every continuously convergent sequence in $Z^{Y}(t)$ converges, the topology need not necessarily be proper.

A rather parallel criterion for admissibility can also be stated. We formulate it now but leave the proof, which resembles that of (2.4), to the reader.

(2.5) THEOREM. Let $Z$ and $Y$ be two spaces. A topology $t$ in $Z^{Y}$ is admissible if and only if for every directed system $\triangle$ and $\triangle$-directed set $\left\{f_{\mu}\right\}$ the convergence $f_{\mu} \rightarrow f$ in $Z(t)$ implies the continuous convergence of $f_{\mu}$ to $f$.

Kuratowski [11] has shown that the idea of continuous convergence can be used to introduce a convergence (in Kuratowski's case $[10], L^{*}$-convergence) in $Z$ provided also $Y$ and $Z$ are $L^{*}$-spaces. The convergence obtained is both admissible and proper, in a suitable sense (see Kuratowski [11]). There is not always a corresponding topology in $Z^{Y}$ associated with this convergence, but the poor showing of topologies in this connection (see (6.01)) seems to commend this step beyond the class of topological spaces, as Kuratowski points out.

3. Comparison of topologies. Since we are going to be concerned with various topologies for $Z^{Y}$ it is natural to recall that there is a useful partial ordering for all the topologies on a fixed fundamental set $E$. For references, see Birkhoff $[5, \mathrm{p} .173]$. 
To define this partial ordering, it is useful to take the attitude that a topology $t$ on a set $E$ is (rather than merely determines) the class of those sets which are open in the topology. Thus a topology is a subset of $2^{E}$, the class of all subsets of $E$. Hence for two topologies $t$ and $u$ on the same set $E$ the set-theoretic statement of inclusion, " $t \subset u$," is meaningful and leads to the following definition.

(3.1) DEFINITION. If $t$ and $u$ are two topologies for a set $E$, we shall write $t \leq u$ or $u \geq t$ or $t$ is smaller than $u$ or $u$ is greater than $t$ when every set open in $t$ is open in $u$, that is, when $t \subset u$.

Notice that the statement " $t$ is smaller than $u$ " is not comparable $w$ ith the statement " $u$ is not greater than $t$," since the former is not intended to exclude the possibility: $t=u$. If $t \leq u$ we shall sometimes call $u$ an expansion of $t$ and $t$ a contraction of $u$. This partial ordering is easily seen to have the property that $t \leq u$ if and only if the identity mapping

$$
E(u) \rightarrow E(t)
$$

is continuous.

Since the class $\tau(E)$ of all topologies is a subset of $2^{E}$, and since the relation " $\leq$ "defined above is just that which is inherited from the natural partial ordering (by inclusion) (see Birkhoff $[5]$ ) in $2^{E}$, we have the following result.

(3.3) THEOREM. The relation " $\leq$ " in the class $\tau(E)$ of topologies on $E$ is a partial ordering.

$\tau(E)$ is not a sublattice of $2^{E}$ because, while $t \cap u$ is always a topology, $t \cup u$ is not always a topology. This does not exclude the possibility that $\tau(E)$ be nevertheless a lattice (see Birkhoff $[5, \mathrm{p} .19]$ ).

(3.4) THEOREM (Birkhoff [4]). $\tau(E)$ is a lattice; that is, for two topologies $t$ and $u$ there is a least upper bound $t \vee u$ and a greatest lower bound $t \wedge u$. In fact, every subset $T$ of $\tau(E)$ has a least upper bound (briefly: "join")

$$
\underset{t \in T}{\vee} t
$$

and a greatest lower bound (briefly: "meet")

$$
\wedge_{t \in T} t
$$

that is, $\tau(E)$ is a complete lattice with greatest and least members.

The greatest lower bound of a class $T$ of topologies has the open sets

$$
\bigcap_{t \in T} t
$$


which are open in all. On the other hand, if we take as a basis the class of sets

$$
U \mathrm{t}
$$$$
t \in T
$$

we obtain a topology which is easily seen to be the least one including all the members of $T$. The discrete topology is the greatest of all. topologies and the trivial topology (only $E$ and the void set are open) is least.

The familiar classes of topologies (for example, Hausdorff, completely regular, normal) are not all well behaved with reference to expansion and contraction (see Hewitt [9]). Since there will emerge some trouble with admissible topologies under the operation "meet," it is only fair to show that things do not go smoothly with every one of the familiar classes of topologies. The following theorem is intended only for orientation. The statement that a property $T$ is preserved under "meeting of two" means that if $t$ and $u$ have property $T$ then so does $t \wedge u$, and so on for the other terms to be used.

(3.5) ТНЕОRЕM. In the lattice of topologies on a set $E$,

(3.51) The Riesz $\left[T_{1}\right]$, Hausdorff $\left[T_{2}\right]$, and Urysohn (see Hewitt [9]) separation properties are each preserved under arbitrary expansions (Hewitt [9]), and hence under joining:

(3.52) Although not preserved under arbitrary expansion (Hewitt [9]), regularity and complete regularity are preserved under joining of two;

(3.53) Riesz separation is preserved under meeting (Birkhoff [4]);

(3.54) Hausdorff and Urysohn separation, regularity, complete regularity, normality, complete normality, and metrizability are not generally preserved under meeting of two.

Proof. Statements (3.51) through (3.53) may be found in the references or easily proved. We content ourselves by supplying an example supporting (3.54).

Let $E$ be any denumerable infinite set, and let $x_{1}, x_{2}$ be a pair of distinct elements of $E$. Consider the topology $t_{1}$ in which any set is open if it either excludes $x_{1}$ or has a finite complement. This (compact) space has all the properties mentioned in (3.54). By interchanging the roles of $x_{1}$ and $x_{2}$ we obtain another topology $t_{2}$. Since $t_{1} \wedge t_{2}$ is a non-Hausdorff Riesz space, all the properties in (3.24) also fail since each guarantees Hausdorff separation when points are closed sets. This completes (3.5).

The result (3.54) just obtained entitles one to consider that perhaps the comparison of topologies based on (3.1) is not the most satisfactory one possible. However, no other generally applicable definition of ordering seems to have been proposed anywhere. 
We now apply these ideas to topologies on a function space $Z^{Y}$.

(3.6) THEOREM. Let $t$ and $u$ be topologies on $Z^{Y}$. If $t$ is admissible and $u \geq t$ then $u$ is admissible. If $u$ is proper and $t \leq u$ then $t$ is proper.

These facts follow at once from the definition and property (3.2). In particular, admissibility is preserved under joining, and properness is preserved under meeting.

We shall see in (5.1) and (5.2) that the proper topologies form a principal ideal $\left(t_{m}\right)$ in the lattice of all topologies; that is, there exists a topology $t_{m}$ such that $t$ is proper if and only if $t \leq t_{m}$. In particular, they constitute a sublattice. For admissible topologies, there sometimes exists a topology $u$ such that $u \leq t$ precisely for the admissible topologies, but sometimes (see (6.3)) not even $t \wedge u$ is admissible when $t$ and $u$ are.

The general position of the admissible topologies with respect to the proper ones is this:

(3.7) ТНЕ ОRЕM. If $t$ is proper and $u$ is admissible then $t \leq u$.

Proof. Since $u$ is admissible, the mapping

$$
\text { - } \omega: Z^{Y}(u) \times Y \rightarrow Z
$$

is continuous. From the definition of "proper," we obtain

$$
\omega^{+}: Z^{Y}(u) \rightarrow Z^{Y}(t) \text {. }
$$

From (3.2) we conclude that $t \leq u$.

See also (6.01) below.

4. Examples of function spaces. In this section we shall give examples of function spaces, some having a proper topology, and some an admissible topology; we also investigate in some detail a method for introducing topologies in the set $Z^{Y}$. Notice that the discrete topology in the set $Z^{Y}$ is always an (the greatest) admissible topology, and the trivial topology (3.4) in $Z^{Y}$ is always a (the smallest) proper topology. We proceed to less trivial methods for introducing a topology.

(4.01) Definition. Let $A$ and $B$ be subsets of the spaces $Y$ and $Z$ respectively. The symbol $(A, B)$ denotes the set of all $f \in Z^{Y}$ satisfying $f(A) \subset B$.

We utilize this notation to define a class of topologies in $Z^{Y}$ : the $\sigma$-topologies. Let $\sigma$ be an arbitrary covering of $Y$ by open sets; we keep $\sigma$ fixed throughout this discussion. Introduce a topology in $Z^{Y}$ as follows. Let $F$ be any closed set in $Y$ contained in some member of $\sigma$, and $V$ an open set in $Z$. The class of all sets of form $(F, V)$ is taken as a subbasis in $Z^{Y}$.

(4.02) DEFINITION. The topology in $Z^{Y}$ thus determined by $\sigma$ is called the $\sigma-$ topology. 
(4.1) THEOREM. Let $Y$ be regular, $Z$ arbitrary. Then for any $\sigma$, the $\sigma$-topology in $Z^{Y}$ is always admissible.

Proof. We are to show that we have $\omega: Y \times Z^{Y} \rightarrow Z$. Let $f \in Z^{Y}, y \in Y$, and let $W$ be a neighborhood of $f(y)$ in $Z$. Since $Y$ is regular, we can find an open $V$ containing $y$ with closure $V^{-}$in $f^{-1}(W)$ and also in some member of $\sigma$; then $\omega\left(V \times\left(V^{-}, W\right)\right) \subset W$ and $\omega$ is continuous, as was to be shown.

The following fact about $\sigma$-topologies is to be compared with (6.3) below.

(4.11) THEOREM. Let $\sigma_{1}$ and $\sigma_{2}$ be open coverings of $Y$. If $\sigma_{1}$ is a refinement of $\sigma_{2}$ then the $\sigma_{1}$-topology is less than or equal to the $\sigma_{2}$-topology. If $Y$ is regular, the meet of two $\sigma$-topologies is also admissible.

Proof. The first assertion is obvious. It implies the second as follows. Let $\sigma$ be a common refinement of $\sigma_{1}$ and $\sigma_{2}$. When $Y$ is regular, the $\sigma$-topology is admissible, and since

$$
\sigma \text {-topology } \leq \sigma_{1} \text {-topology } \wedge \sigma_{2} \text {-topology, }
$$

the latter is admissible. There is no reason why the latter should be a $\sigma$-topology, of course.

Variants of the $\sigma$-topologies can be found by varying the allowable sets in $Y$, that is, by permitting open, or arbitrary, subsets of members of $\sigma$ to be used in the definition of the subbasis. Although these variants of $\sigma$-topologies are also always admissible (when $Y$ is regular) there is a reason for preferring the $\sigma$-topologies. To see this, we first remark that the existence of a proper admissible topology in $Z^{Y}$ is a desirable property. For example, it is easily seen that with such a topology, the homotopy of two maps $Y \rightarrow Z$ is equivalent with their being joined by an arc in the functional space. Now, when $Y$ is regular, it is easy to see that the $\sigma$-topology is always less than or equal any of its variants, so that the former is "nearer" to the proper topologies than any of the latter. For this reason, the $\sigma$-topologies appear better suited to our work.

We shall now introduce a class of topologies including the class of $\sigma$-topologies. Let $Y$ and $Z$ be as before and let a family $\{A\}$ of subsets of $Y$ be given. Taking the family of sets $(A, W)$ (see (4.01)), where $W$ is open in $Z$ and $A$ belongs to $\{A\}$, as a subbase in $Z^{Y}$ we obtain a topology.

(4.2) Definition. The topology described above is called the $\{A\}$-open topology. Any such topology will be called an $S$-topology, or set-open topology. The space $Z^{Y}$ with the $\{A\}$-open topology will be written $Z^{Y}(S:\{A\})$.

One reason for still limiting $W$ to open subsets of $Z$ in $(A, W)$ is that in this 
way we can be sure that if we consider only the class of constant functions, it will be homeomorphic to $Z$.

The next result shows how a large class of proper topologies may be obtained. (Recall that $\sigma$-topologies provide a way of obtaining admissible topologies.)

(4.21) THEOREM. Let $Z$ and $Y$ be two spaces. If all the sets in $\{A\}$ are compact, then the $\{A\}$-open topology in $Z^{Y}$ is proper.

Proof. Suppose we have $g: X \times Y \rightarrow Z$; to prove $g^{*}$ continuous, it is enough to show that given any subbasic open set $(A, V) \ni g *\left(x_{0}\right)$, there is a neighborhood $U$ of $x_{0}$ with $g^{*}(U) \subset(A, V)$. Using the continuity of $g$ and the definition of $g^{*}$, we see that from $x_{0} \times A$ being contained in the open set $g^{-1}(V)$ we have to conclude that $U \times A \subset g^{-1}(V)$ for some neighborhood $U$ of $x_{0}$. To do this, for each $\left(x_{0}, y\right)$ in $x_{0} \times A$ we find a set $W_{y}$ open in $X \times Y$ with $\left(x_{0}, y\right) \in \mathbb{W}_{y} \subset g^{-1}(V)$; this gives a covering of $x_{0} \times A$, and the compactness of $x_{0} \times A$ allows us to extract a finite covering. The intersection of the projections of the sets of this finite covering on $X$ gives an open $U$ containing $x_{0}$ and clearly $U \times A \subset g^{-1}(V)$.

On the basis of this theorem, an important special case of the set-open topologies is singled out: the case where $\{A\}$ is the collection of all the compact subsets of $Y$ (see Arens [2], Fox [7]). We will call this special case, for ready reference, the $k$-topology. For separation properties of the $k$-topology, see Arens [2]. For example, if $Z$ is a Hausdorff space, the $k$-topology is a Hausdorff topology. It is evident that the $k$-topology is the greatest set-open proper topology based on compact sets.

The proof of the properness of a set-open topology contains essentially the following question: What conditions on the sets $\{A\}$ insure that, for every $X$, an open $V$ in $X \times Y$ containing $x_{0} \times A$ also contains an "open tube" $U \times A$ ( $U$ a neighborhood of $x_{0}$ in $\left.X\right)$ ? With this observation, we are ready to approach the problem: Which of the set-open topologies are proper? Our procedure enables us to answer a more inclusive question: What conditions on the class $\{A\}$ follow from the assumption that the $\{A\}$-open topology is $\leq$ every admissible topology? (See (3.7).) A sufficient condition has been given in (4.2); we have several necessary conditions, but have not found both necessary and sufficient conditions, except in isolated instances.

We first treat the special case of real-valued functions.

(4.3) Тнеовем. Let $Y$ be a completely regular space, and $E_{1}$ the Euclidean line. If the $\{A\}$-open topology in $E_{1}^{Y}$ is $\leq$ every admissible topology, then the sets of $\{A\}$ must all have compact closure.

Proof. Let $B$ be any set of $\{A\}$ and $\sigma:\{V\}$ an arbitrary covering of the closure $B^{-}$of $B$. We are to show that we can extract a finite covering of $B^{-}$.

Let $f$ be the constant function 0 in $E_{1}^{Y}$. Then $f \in(B, W)$, where $W$ is the complement of 1 in $E_{1}$. Now form the $\sigma$-topology based on the covering of $Y$ by the 
sets $\{V\}$ together with the complement of $B^{-}$. By (4.11) this topology is admissible, and by hypothesis there exists a neighborhood

$$
U=\left(C_{1}, C_{2}, \cdots, C_{n} ; W_{1}, W_{2}, \cdots, W_{n}\right)
$$

in $Z^{Y}(\sigma$-topology) such that $f \in U \subset(B, W)$. Let $C$ denote the closed union of $C_{1}, \cdots, C_{n}$. If $C$ does not contain $B^{-}$, there is a point $b$ in $B^{-}$which is not in $C$, and which hence has a neighborhood $V$ not meeting $C$; since $b \in B^{-}$, it follows that $V$ contains some point $b^{\prime}$ in $B$ not in $C$. Construct a continuous real-valued function $r$ with $r\left(b^{\prime}\right)=1$ and $r(y)=0$ for $y \notin V$. It is clear that $r \in U$ since it coincides with $f$ on $C$, but evidently $r \notin(B, W)$. Hence, $B^{-}$is contained in $C$. Let $V_{1}, \cdots, V_{n}$ be sets of the covering $\sigma$ containing the closed sets $C_{1}, \cdots, C_{n}$ respectively; then $B^{-}$is contained in the union of the former. Hence, $B^{-}$is compact, as was to be shown.

It is evident that a similar theorem holds for mappings of a completely regular space $Y$ into any space $Z$ that contains at least one non-degenerate arc. Thus an application of the special case (4.3) yields the same conclusion in many more general cases.

(4.31) THEOREM. Let $Y$ be a completely regular space, and $Z$ a space containing a non-degenerate arc. A necessary and sufficient condition that a set-open topology based on closed sets be $\leq$ every admissible topology is that it be a proper topology. (3.7).

Proof. The necessity stems from (4.3) and (4.21). The sufficiency arises from

(4.311) CoRollaRY. Let $Y$ be a completely regular space, and $Z$ a space containing a non-degenerate arc. A set-open topology based on closed sets is proper if and only if all the sets are compact.

The following concept is useful in the further investigation of $\{A\}$ when the $\{A\}$-open topology is proper. Let $B$ be a subset of $Y$. A point $y_{0}$ of $Y$ is inessential to $B$ if, for every $f: Y \rightarrow E_{1}$ there exists a $y$ in $B, y \neq y_{0}$, such that $f(y)=f\left(y_{0}\right)$. Note that in a metric space $Y$ no point is inessential to any $B$. In a completely regular space, $y_{0}$ is essential (that is, not inessential) to $B$ if and only if $y_{0}$ is a $G_{\delta}$-set relative to $B$.

(4.4) THEOREM. Let $Y$ be an arbitrary space, $Z$ a space containing a nondegenerate arc. If the $\{A\}$-open topology in $Z^{Y}$ is $\leq$ every admissible topology, then each set $A$ of $\{A\}$ must contain all points of its closure which are essential to $A$.

Proof. One may regard $E_{1}$ as embedded in $Z$. Let $A$ belong to $\{A\}$, and let $Y \in A^{-}-A$. Suppose $y_{0}$ is essential to $A$. Then there exists an $f: Y \rightarrow E_{1}$ with 
$f(y) \neq f\left(y_{0}\right)$ for every $y$ in $A$. Let us take $f\left(y_{0}\right)=0$.

Select $X=E_{1}$ and define $g: E_{1} \times Y \rightarrow E_{1}$ by the condition $g(x, y)=x+f(y)$. We can now define an admissible topology $t$ in $Z^{Y}$ as follows: (a) the set

$$
G=\left\{g *(x) ; x \in E_{1}\right\}
$$

is open and homeomorphic to $E_{1}$; (b) all other elements are isolated. This topology is clearly admissible, due to the continuity of $g$. By hypothesis there must exist a set $U$ open in $Z^{Y}(t)$ with $f \in U \subset(A, W)$; and due to the definition of $t$, this $U$ is (or at least contains) the image of some interval $(-e, e), e>0$. Now, since $y_{0}$ is a limit point of $A$, and $f$ is continuous, there must exist a $y_{0}$ in $A$ with $-e<f\left(y_{0}\right)<e$. Construct $f_{1}=g^{*}\left[-f\left(y_{1}\right)\right]$. This $f_{1}$ belongs to $U$, but it does not belong to $(A, W)$ since $f_{1}\left(y_{1}\right)=-f\left(y_{1}\right)+f\left(y_{1}\right)=0$. This is a contradiction, and shows that $y_{0}$ is inessential to $A$. This proves (4.4).

We give an example to show that the sets $\{A\}$ on which a proper $S$-topology is based need not be closed. Let $Y$ be any uncountable set in which all points are declared open sets except one, $y_{0}$, whose neighborhoods are defined as the complements of finite sets excluding $y_{0}$. Introduce a set-open topology $t$ into $E_{1}{ }_{1}^{Y}$ based on the non-closed set $A=Y-y_{0}$. Note that $g \in\left(Y-y_{0}, W\right)$ if and only if $g \in(Y, W)$, since otherwise $g$ would assume a value at $y_{0}$ different from all its other values, and $y_{0}$ would be a $G_{\delta}$. Thus this topology is the same as that based on $A_{1}=Y$, which is proper, by (4.3), since $A_{1}$ is compact.

With the aid of this Theorem (4.4), one can refine the results of (4.31) and (4.311). We state the result but leave the proof to the reader.

(4.41) THEOREM. Let $Y$ be a completely regular space in which every point is $a G_{\delta}$, and $Z$ any space that contains a non-degenerate arc. A set-open topology in $Z^{Y}$ is proper if and only if it is based on sets that are all compact. A necessary and sufficient condition that a set-open topology in $Z^{Y}$ be proper is that it be $\leq$ every admissible topology.

If a simple condition be satisfied by $Z$, we lose no proper set-open topologies by limiting ourselves to $\{A\}$-open topologies where every $A$ is compact. This is shown in the next theorem.

(4.5) THE OREM. Let $Y$ be a completely regular space, $Z$ a metric space containing a non-degenerate arc. If an $\{A\}$-open topology in $Z^{Y}$ is $\leq$ every admissible topology, then it is equivalent to the set-open topology based on the compact sets $\left\{A^{-}\right\}$.

Proof. We have, by (4.3), that all the sets $A^{-}$are compact; on the basis of (4.4) every $A$ contains all points of $A^{-}$that are essential to $A$. Let $A_{0}$ be the set of points of $A^{-}$inessential to $A$; then $A \cup A_{0}=A^{-}$. The theorem will be proved when we show that, for the subbasic open sets, we have $\left(A \cup A_{0}, \mathbb{W}\right)=(A, \mathbb{W})$. The 
inclusion $\left(A \cup A_{0}, \mathbb{W}\right) \subset(A, \mathbb{W})$ is evident. To prove also $(A, \mathbb{W}) \subset\left(A \cup A_{0}, \mathbb{W}\right)$ we need only show that $f \in(A, W)$ implies $f\left(a_{0}\right) \in \mathbb{W}$ for every $a_{0} \in A_{0}$. To this end denote by $d$ the metric in $Z$, and set $F(y)=d\left(f(y), f\left(a_{0}\right)\right)$; then we have

$$
F: Y \rightarrow E_{1},
$$

and since $a_{0} \in A_{0}$, this means there must be a point $a \in A$ with $F(a)=F\left(a_{0}\right)=0$; this in turn implies that $f\left(a_{0}\right)=f(a) \in \mathbb{W}$, finishing the proof as indicated. (All that is really needed for this theorem is that (a) $Z$ be completely regular, (b) every point of $Z$ be a $G_{\delta}$, and (c) $Z$ contain a non-degenerate arc.)

If we specialize $Z$ instead of $Y$, we get a more complete converse to (4.2). Let us take $Z$ to be the Sierpinski space consisting of two points, which we call 0 and 1 , with the empty set, the entire space, and the point 0 as the only open sets.

(4.6) The овем. Let $Y$ be an arbitrary space, $S$ the Sierpinski space. A necessary and sufficient condition that an $\{A\}$-open topology in $S^{Y}$ be $\leq$ every admissible topology is that all the sets of $\{A\}$ be compact.

Proof. The sufficiency follows from (4.2). We need only prove the necessity. Let $B$ be an arbitrary set of the collection $\{A\}$ and $\left\{V_{\beta}\right\}$ an arbitrary covering of $B$. We shall reduce $\left\{V_{\beta}\right\}$ to a finite covering. Let $V$ be the union of all the $V_{\beta}$.

Let $k \in S^{Y}$ be the function which is 0 precisely on $V$. Introduce an admissible topology $t$ in $S^{Y}$ as follows: (a) All elements of $S^{Y}$ except $k$ are isolated, (b) the neighborhoods of $k$ are of form $\left(V_{1} \cup V_{2} \cup \cdots \cup V_{\beta}, 0\right)$ where the $V_{i}$ are open sets with $V_{i} \subset V_{\beta_{i}}$. In fact, $t$ is admissible, as is not hard to verify. The hypothes is then gives us a neighborhood in $t$ with

$$
k \in\left(V_{1} \cup V_{2} \cup \cdots \cup V_{n}, 0\right) \subset(B, 0) .
$$

Selecting $V_{\beta_{i}} \supset V_{i}$, we form their union $G$. This set covers $B$; for otherwise, if $g$ is the function vanishing precisely on $G$ we have $g \in\left(V_{1} \cup \cdots \cup V_{n}, 0\right)$ and $g \notin(B, 0)$, a contradiction. Hence $B$ is compact, proving (4.6).

We now turn to the admissible case, and seek conditions under which a setopen topology is admissible. For convenience we make another definition. A family of sets $\{A\}$ is a regular family in $Y$ if, given any $y$ in $Y$ and neighborhood $U$ of $y$, there exists an $A$ in $\{A\}$ contained in $U$ and containing $y$ in its interior.

This concept permits the following statement.

(4.7) THEOREM. Let $Z$ and $Y$ be arbitrary spaces. A set-open topology in $Z^{Y}$ based on a regular family of sets is always admissible.

Proof. We are to show that we have $\omega: Y \times Z^{Y} \rightarrow Z$. Let $f \in Z^{Y}, y \in Y$ and $W$ a neighborhood of $f(y)$; then $f^{-1}(W)$ is open in $Y$, and $y \in f^{-1}(W)$; by regularity of the family $\{A\}$ we can find an $A$ with $y \in$ int $A, A \subset f^{-1}(W)$. It is clear that $\omega\left[\right.$ int $\left.A \times\left(A_{1}, W\right)\right] \subset W$, and so $\omega$ is continuous. 
This theorem has two interesting consequences, the first of which has been known for a long time; see Fox [7] and Arens [2].

(4.71) THEOREM. Let $Y$ be a regular locally compact space, and $Z$ arbitrary. Then the k-topology (see (4.2)) in $Z^{Y}$ is admissible and proper.

Proof. The totality of all compact subsets of $Y$, since $Y$ is locally compact and regular, forms a regular family.

A corollary of (4.71) particularly useful in discussions of homotopies is the following.

(4.72) Cor Ollary (Fox [7]). Let $Y$ be regular, and let $X$ and $Y$ both satisfy the first axiom of countability. Then $g: X \times Y \rightarrow Z$ is equivalent with

$$
g^{*}: X \rightarrow Z^{Y}(k) \text {, }
$$

for any $Z$.

Proof. One half of the result comes from (4.2); we prove $g^{*}: X \rightarrow Z^{Y}(k)$ implies $g: X \times Y \rightarrow Z$. Note that (4.71) implies $g$ is continuous on all sets of form $A \times Y$ where $A$ is a compact subset of $X$. In particular, $g$ is sequentially continuous, and with our hypothesis this implies that $g$ is continuous.

5. The proper topologies. The situation of the proper topologies in the class of all topologies in a class $Z^{Y}$ is a particularly simple one: with the partial ordering of (3.1), they form an ideal with a (smallest and a) greatest element. We establish first the completeness of the class of proper topologies.

(5.1) LEMMA. Let $Z$ and $Y$ be arbitrary spaces; let $\left\{t_{\alpha}\right\}$ an arbitrary collection of proper topologies in $Z Y$. Then $\wedge_{\alpha} t_{\alpha}$ and $\vee_{\alpha} t_{\alpha}$ are also proper topologies in $Z^{Y}$.

Proof. That $\Lambda_{\alpha} t_{\alpha}$ is a proper topology is immediate from (3.6). To prove the remaining part, suppose we have $g: X \times Y \rightarrow Z$; we are to show that we have $g^{*}: X \rightarrow Z^{Y}\left(\vee_{\alpha} t_{\alpha}\right)$. Select an open set $U$ in $Z^{Y}\left(\vee_{\alpha} t_{\alpha}\right)$; since it is sufficient to consider only the subbasic open sets, this selected set can be assumed open in some topology $t_{\beta}$. Since $t_{\beta}$ is proper, the inverse image of $U$ under $g^{*}$ is open in $X$. Hence $g^{*}$ is continuous, as was to be shown.

Since an application of (5.1) gives a greatest and a least proper topology, we may reformulate $(5.1)$ in the following way.

(5.2) THEOREM. Let $Z$ and $Y$ be arbitrary spaces. With the partial ordering of (3.1), the proper topologies in $Z^{Y}$ form an ideal with a greatest element.

The least proper topology is, of course, the trivial topology. The (unique) greatest proper topology $t_{m}$ can be characterized as follows: let $\left\{t_{\alpha}\right\}$ be the collection of all the proper topologies in $Z^{Y}$; then $t_{m}=V_{\alpha} t_{\alpha}$. We have been unable 
to characterize this greatest proper topology more directly in terms of the topological structures of $Z$ and $Y$. So far as its properties are concerned, since $t_{m} \geq k$ topology, all properties of the $k$-topology invariant under expansion (such as Hausdorff separation, disconnection) are inherited by $t_{m}$. A method for obtaining the greatest proper topology that sometimes works will be given in Section 6; we merely remark here that a proper admissible topology in $Z^{Y}$ is the greatest proper topology.

The problem initiating this paper was to determine the status of the $k$-topology. From (4.71) and the above remark, we note that if $Y$ is locally compact, the $k$ topology is in fact the greatest proper topology in $Z^{Y}$. We ask then if the $k$-topology has any distinguished role in the hierarchy of proper topologies for $Z^{Y}$. Theorems (5.4) and (5.5), below, will give a reason why the $k$-topology is a convenient topology to be used in function spaces. However, it is not distinguished by being always the greatest proper topology in $Z^{Y}$. We now present an example.

(5.3) ТнЕОRЕм. Let $Z$ be the unit interval $[0,1]$ in $E_{1}$. Then there exists a completely regular space $Y$ such that the $k$-topology in $Z^{Y}$ is not the greatest proper topology.

Proof. Let $Y$ be the set of all ordered pairs of positive integers, and one additional element which we will call $\infty$. The topology in $Y$ is obtained by taking each pair $(i, j)$ as an isolated point, and the neighborhoods of $\infty$ to be all sets obtained as follows: if $N, J_{N+1}, J_{N+2}, \cdots$ is any collection of integers, the set

$$
V=\left\{(i, j) ; i>N \text { and } j>J_{i}\right\}
$$

is a neighborhood of $\infty$. We remark that, in this space $Y$, all the compact sets are finite sets; see Arens [3, p. 234].

Define a function $f_{n}: Y \rightarrow Z, f_{n}(i, j)=0$ or 1 according as $i \neq n$ or $i=n$, and $f_{n}(\infty)=0$. Expand the topology of $Z^{Y}(k)$ by declaring the set $B=\left\{f_{1}, f_{2}, \cdots\right\}$ closed, thus obtaining a topology $k^{+}$in $Z$. We note first that $k^{+}>k$ since $f_{n} \rightarrow 0$ in the $k$-topology, but not in the $k^{+}$-topology.

The theorem will be proved when we show that $k^{+}$is a proper topology in $Z^{Y}$. To this end, let $g_{\mu}$ converge continuously to $g$ (see (2.4)); we are to prove $g_{\mu} \rightarrow g$. Our proof breaks into two cases.

Case 1: $g \neq 0$. Since $Z^{Y}(k)$ is a Hausdorff space (see (4.3)), if $g \neq 0$, we can find a $k$-neighborhood $U$ of 0 that excludes the sequence $B$, because $f_{n} \rightarrow 0$ implies that 0 is the only limit point of $B$ in $Z^{Y}(k)$. Since the topologies of $Z^{Y}\left(k^{+}\right)$ and $Z^{Y}(k)$ coincide at all points $g \neq 0$, and $k$ is a proper topology, this means $g_{\mu}$ is ultimately in $U$, and so converges to $g$ in $k$ and in $k^{+}$.

Case 2: $g=0$. Let $U^{+}$be a neighborhood of 0 in $k^{+}$. Then $U^{+}=U-B$ ( $U$ open in $k$-topology), and $g_{\mu}$ is again ultimately in $U$. Let us assume that nevertheless $g_{\mu} \nrightarrow 0$ in $k^{+}$. Then we must have $g_{\mu} \in B$ cofinally; that is, given 
$\mu$, there exists a $\mu^{\prime} \geq \mu$ with $g_{\mu^{\prime}} \in B$. The $g_{\mu^{\prime}}$ still converge continuously to 0 . Hence there is a neighborhood $V$ of $\infty$ (say the one described in the first paragraph) and a $\mu_{0}$ such that for $\mu^{\prime} \geq \mu_{0}$, we have $g_{\mu^{\prime}}=0$ on $V$. But since $g_{\mu^{\prime}} \in B$, we have $g_{\mu^{\prime}}=f_{n\left(\mu^{\prime}\right)}$; and since $g_{\mu^{\prime}}=f_{n}\left(\mu^{\prime}\right)=0$ on $V$ for $\mu^{\prime} \geq \mu_{0}$, we would have $1 \leq n\left(\mu^{\prime}\right) \leq N$ for $\mu^{\prime} \geq \mu_{0}$. Since the $f_{n}\left(\mu^{\prime}\right)$ converge continuously to 0 , they converge also in the $k$-topology (see (2.4)). Hence there exists a $\mu_{1}$ such that $\mu \geq \mu_{1}$ implies $f_{n(\mu)}(i, 1)=0$ for $i=1,2, \cdots, N$; this means that for $\mu^{\prime} \geq \mu_{0}$, $\mu^{\prime} \geq \mu_{1}$, we have $g_{\mu^{\prime}}=0=f_{n}\left(\mu^{\prime}\right)$. Thus the $g_{\mu^{\prime}}$ are finally all 0 , contradicting that they all lie on $B$. Hence, $g_{\mu} \rightarrow 0$ in $k^{+}$. This concludes the proof of the fact that $k^{+}$is proper.

If we do not require $Y$ to be a completely regular space, then a construction of a proper topology greater than the $k$-topology becomes simpler. We append such an example for later use.

(5.31) LEMMA. Let $Y$ be a completely regular space, satisfying the first axiom of countability, and $Z$ the unit interval in $E_{1}$. Let $Y^{\sim}$ be an expansion of the topology of $Y$ in such a way that the sets $Z^{Y}$ and $Z^{Y^{\sim}}$ are the same. Then for any space $X, g: X \times Y^{\sim} \rightarrow Z$ implies $g: X \times Y \rightarrow Z$.

Proof. We first establish the following results.

(5.311) A point $y$ in $Y^{\sim}$ has a basis of neighborhoods of the form $V-D^{\sim}$, where $V$ is open in $Y, D^{\sim}$ is closed in $Y^{\sim}$, and $V \cap D^{\sim}$ has no interior in $Y$.

To see this, note that any neighborhood of $y$ in $Y^{\sim}$ has the form $Y^{\sim}-E^{\sim}$, where $E^{\sim}$ is closed in $Y^{\sim}$. Suppose now that the interior $I$ of $E^{\sim}$ in $Y$ has $y$ as limit point. Pick a basis $V_{1}, V_{2}, \cdots$ of $y$ in $Y$ such that

$$
V_{1} \supset V_{2}^{-}, V_{2} \supset V_{3}^{-}, \cdots \text {, }
$$

in such a manner that $I \cap\left(V_{n}-V_{n+1}^{-}\right) \neq 0$. Define $f_{n}: Y \rightarrow Z$ by

$$
f_{n}(y)=\left\{\begin{array}{l}
1 \text { at some point of } I \cap\left(V_{n}-V_{n+1}^{-}\right) \\
0 \text { for } y \notin I \cap\left(V_{n}-V_{n+1}^{-}\right) .
\end{array}\right.
$$

Then $f=\Sigma_{n} f_{n}$ is a continuous on $Y$ except at $y . \operatorname{In} Y^{\sim}$, however, it is continuous even at $y$ because $f=0$ on $Y^{\sim}-E^{\sim}$. The real-valued continuous functions being the same, this can only happen if $I$ does not have $y$ as a limit point in $Y$. Hence, we can pick $V$ so that $V \cap E^{\sim}$ has no interior in $Y$, as was to be shown.

(5.312) $g: X \times Y^{\sim} \rightarrow Z$ implies $g: X \times Y \rightarrow Z$.

With the notations of (5.311), $g: X \times Y^{\sim} \rightarrow Z$ implies that there exists a neighborhood $U$ of $x_{0}$ and a $V-D^{\sim}$ containing $y_{0}$ such that

$$
\left|g(x, y)-g\left(x_{0}, y_{0}\right)\right|<\epsilon
$$


for $(x, y) \in U \times\left(V-D^{\sim}\right)$. Now, each point of $V \cap D^{\sim}$ is a limit point of $V-D^{\sim}$; so if $y \in\left(V \cap D^{\sim}\right)$, there exists a sequence $\left\{y_{\mu}\right\}$ with $y_{\mu} \in\left(V-D^{\sim}\right)$ and $y_{\mu} \rightarrow y$ in $Y$. Since $g(x, \cdot)$ is still continuous on $Y$, we have $\left|g(x, y)-g\left(x_{0}, y_{0}\right)\right| \leq E$ and therefore $g: X \times Y \rightarrow Z$, as desired.

(5.32) THEOREM. Let $Y$ be a completely regular space, satisfying the first axiom of countability, and let $Z$ be the unit interval of $E_{1}$. Let $Y^{\sim}$ be an expansion of the topology of $Y$ that introduces no new continuous real-valued functions. Let $Z Y^{\sim}\left(k^{\sim}\right)$ be the functional space with the $k$-topology, and let $Z^{Y^{\sim}}{ }^{(k)}$ be the same functional space with the $k$-topology of $Z^{Y}$. Then $k>k^{\sim}$, and $k$ is also proper. Hence, the k-topology in $Z^{\sim}$ is not the greatest proper topology.

Proof. It is not hard to verify that in fact $k>k^{\sim}$. To see that $k$ is also proper, note that $g: X \times Y^{\sim} \rightarrow Z$ implies $g: X \times Y \rightarrow Z$, which yields

$$
g^{*}: X \rightarrow Z Y(k)=Z^{Y^{\sim}}(k) \text {, }
$$

as was to be shown.

An example of such a space is exhibited in (6.21) below.

The position of the $k$-topology in the proper topologies for $Z^{Y}$ can now be somewhat clarified; and a reason for its utility will appear from the following sequence of theorems.

(5.4) THEOREM. Let $Y$ be a completely regular space, and $Z$ an arbitrary space. Then the $k$-topology is the greatest of the proper set-open topologies based on closed sets.

(5.5) THEOREM. Let $Y$ be a completely regular space, $Z$ a metric space containing a non-degenerate arc. Then the k-topology is the greatest of the proper set-open topologies.

The proofs of these theorems are immediate from (4.311) and (4.5).

(5.51) COROLLARY. Let $Y$ be a completely regular space, and $Z$ a metric space containing a non-degenerate arc. If the k-topology is not the greatest proper topology, then the greatest proper topology is not a set-open topology.

From consideration of the results in (3.6) it is clear that, if a proper admissible topology exists, there must be a delicate balancing of open sets. In general, there is no such topology (see (6)). But we now show there can never be more than one, if any.

(5.6) THE OREM. Let $Z$ and $Y$ be arbitrary spaces. If $t$ is a proper admissible topology in $Z^{Y}$, then $t$ is unique. That is, there is no other proper admissible topology except $t$. 
Proof. Let $s$ be another proper admissible topology. Since $s$ is proper and $t$ is admissible, by (3.6), we have $s \leq t$. Reversing the roles of $s$ and $t$, we get also $t \leq s$. Hence, $t=s$.

Some of the considerations of this section can be applied to more general situations in which topologies for a set $X$ are considered, there being no function space in the picture. The following generalization of (5.1) is related to a construction of Choquet's [6, p. 85].

(5.7) LEMMA. Let L be a "notion of convergence," that is, a rule which assigns, to some directed sets in $X$, a point. Let a topology $t$ for $X$ be called "L-proper" if whenever L assigns $x$ to $\left\{x_{\mu}\right\}$ then $x_{\mu} \rightarrow x$ in $X$. Then, if $T$ is any family of L-proper topologies, the topology

$$
t^{-}=\underset{t \in T}{\vee} t
$$

is also L-proper.

Proof. Let $L$ assign $x$ to $\left\{x_{\mu}\right\}$. Let a neighborhood $U$ of $x$ in $X\left(t^{-}\right)$be given. There exist $t_{1}, \cdots, t_{n}$ in $T$ such that $U=U_{1} \cap \cdots n U_{n}$, where $U_{i}$ is open in $X\left(t_{i}\right)$. Hence there is some $\mu_{i}$ such that the relation $\mu \geq \mu_{i}$ implies $x_{i} \in U_{i}$. Select $\mu_{0} \geq \mu_{1}, \cdots, \mu_{n}$. For $\mu \geq \mu_{0}$, we have $x_{\mu} \in U$. Thus $t^{-}$is proper.

To obtain (5.1), let $L$ be the notion of continuous convergence, and let $T$ be the class of all $L$-proper topologies.

One may also define what is meant by an " $L$-admissible" topology. A topology $t$ for $X$ is $L$-admissible if whenever $x_{\mu} \rightarrow x$ in $X$, then $L$ assigns $x$ to $\left\{x_{\mu}\right\}$. The next Section shows that the $L$-admissible topologies do not always have the property dual to that established for the $L$-proper topologies in (5.7), not even when $T$ is limited to finite sets.

6. Admissible topologies. The $\sigma$-topologies provide many examples of admissible topologies. We shall now see that proper admissible topologies are scarce, and that the hierarchy of admissible topologies rarely forms a lattice.

(6.01) ThEOREM. A proper admissible topology for $Z^{Y}$ is both the greatest proper topology and the least admissible topology.

Proof. The proof rests on the fact that any proper topology is smaller than or equal to any given admissible topology (see (3.7)).

One conceivable way of determining whether there is a proper admissible topology is to examine the greatest proper topology itself. It is unique and is admissible if and only if there exists a proper admissible topology. A direct examination of the greatest proper topology seems rather cumbersome. The following partial converse to (5.71) shows that, under fairly general conditions, when $Y$ is not locally compact, there is no proper admissible topology. 
(6.1) THEOREM (Fox [7, Theorem 3]). If $Y$ is separable and metrizable and $Z$ is the real line, and if there exists a proper admissible topology, then $Y$ is locally compact.

Now (6.1) is, by (6.01), immediately deducible from the following somewhat stronger result.

(6.2) THEOREM (Arens [2, Theorem 3]). If $Y$ is a completely regular space and $Z$ is the real interval $[0,1]$, and if there exists a least admissible topology for $Z^{Y}$, then $Y$ is locally compact.

In particular, the "separable and metrizable" in (6.1) can be replaced by "completely regular." As to the necessity of complete regularity in (6.2): a locally compact Hausdorff space must be completely regular. However, this justification for assuming $Y$ to be completely regular is not as convincing as is the following example.

(6.21) THEOREM. There exists a non-locally-compact Hausdorff space $Y$ such that $Z^{Y}$ can be given a least admissible topology, where $Z$ is the real interval $[0,1]$.

Proof. Let $Y_{0}$ be the real interval $[0,1]$ with the ordinary topology. Let $y$ be the interval $[0,1]$ with the topology generated by the following subbasic open sets (cf. Alexandroff-Hopf $[1, \mathrm{p} .31])$ : first, the complement of the set $D$, where is the set of numbers $1 / n(n=1,2, \cdots)$; second, the open sets of $Y_{0}$. Thus $Y$ differs from $Y_{0}$ only at 0 , but the mutilation at 0 is enough to make $Y$ an irregular Hausdorff space. Hence it cannot be locally compact. The remaining part of the argument hinges on the fact that a function $f:[0,1] \rightarrow Z$ is continuous on $Y$ if and only if it is continuous on $Y_{0}$. We leave the proof of this to the reader. (This in itself implies that $Y$ is not completely regular.) Let $\{A\}$ be the class of sets which are compact in $Y_{0}$. In $Z^{Y}$ these determine a set-open topology (see (4.2)) which we shall call the $k_{0}$-topology. It is clearly admissible, by (4.7). Let $t$ be any other admissible topology for $Z^{Y}$. Suppose $f \in Z^{Y}$ and let $\left(A, W_{1}\right)$ be a neighborhood of $f$ in $Z^{Y}\left(k_{0}\right)$. Now the image $f(A)$ is surely compact in $Z$ so that we can find an open set $W$ in $Z$ such that $f(A) \subseteq W$ and $W^{-} \subset W_{1}$. For each $y$ in $A$ there is a neighborhood $V_{1}(y)$ of $y$ and a neighborhood $U(f, y)$ of $f$ in $Z Y(t)$ so that $z \in V_{1}(y)$ and so that the relation $g \in U(f, y)$ implies $g(z) \in \mathbb{W}$. Let $V(y)$ be the interior of the closure of $V_{1}(y)$. It is easy to see that $g(z) \in W_{1}$ for $z \in V(y)$ and $g$ as before. Since each $V(y)$ is open in $Y_{0}$, where $A$ is compact, we can find $y_{1}, \cdots, y_{n}$ such that

$$
A \subset V\left(y_{1}\right) \cup \cdots \cup V\left(y_{n}\right)
$$

Let $U(f)=U\left(f, y_{1}\right) \cap \cdots \cap U\left(f, y_{n}\right)$. It is easy to see that $U(f)$ is contained in $\left(A, W_{1}\right)$ (see Arens $\left.[2, \mathrm{p} .482]\right)$. We infer from this that $t \geq k_{0}$. 
The complete regularity is used in the proof of (6.2) (see Arens [2, p.483]) in constructing an element of $Z^{Y}$ which distinguishes a closed set from a disjoint point. Hence the proof may be duplicated in any other case where such separation by continuous functions is always possible.

An application, rather than extension, of (6.2) shows that in (6.2) the space $Z$ may be taken as any $T_{0}$-space containing a non-degenerate arc. Thus we obtain a result when $Z$ is the Sierpinski space $\{0,1\}$. However, in this particular case the proof of (6.2) can be adapted so as to give a still better result: complete regularity is relaxed to regularity. We state the result but leave the proof to the reader.

(6.25) THEOREM. If $Y$ is a regular space and $Z$ is the Sierpinski space (see (4.6)), and if there exists a least admissible topology for $Z^{Y}$, then $Y$ is locally compact.

Returning to the remark just made about having an arc in $Z$, we wish to show that this requirement cannot be simply omitted. Let us consider an extreme example in which $Y$ is connected but $Z$ is totally disconnected. Then $Z^{Y}$ consists only of constant functions and can hence be given the topology of $Z$, which is both proper and admissible, regardless of any other properties of $Y$.

Theorem (6.2) says that when $Y$ is completely regular, and $Z$ is $[0,1]$ but $Y$ is not locally compact, then there is at least one class $a$ of admissible topologies whose greatest lower bound is not admissible. The class $a$ which (6.2) exhibits for this purpose is a large one-in fact the largest possible. One might ask whether any two admissible topologies have an admissible "meet" topology (greatest lower bound) (see (3.4)), especially since we know of an extensive class of admissible topologies (see (4.11)) for which the meet of two is always admissible. The following theorem shows that the answer is "no" for any metric non-locally compact space $Y$. We define a Fréchet-compact set to be one in which every infinite subset has a limit point.

(6.3) THEOREM. Let $Y$ be a completely regular Hausdorff space in which each point has a countable basis, and let $Z$ be the real line or the interval $[0,1]$.If the meet of every pair of admissible S-topologies for $Z^{Y}$ is admissible then $Y$ is locally Fréchet-compact.

Proof. Suppose $Y$ is not locally Fréchet-compact. One can then find a point $y_{0}$ for which one can construct a basis $V_{1} \supset V_{2} \supset V_{3} \supset \cdots$, and a sequence of infinite sets $r_{1}, r_{2}, r_{3}, \cdots$, none of which has a limit point and such that $r_{n}$ is contained in $V_{n}-V_{n+1}$. Break each $r_{n}$ into disjoint infinite subsets $s_{n}, t_{n}$. Let $r$ be the union of $r_{1}, r_{2}, r_{3}, \cdots$. A set $A$ will be called an $R$-set if $A^{-}$intersects $r$ in but a finite set. Let $B_{n}$ be any open subset of $V_{n}-V_{n+1}$ containing all of $t_{n}$ and no points of $s_{n}$, and let $A_{n}$ be any $R$-set. Then let $\left(V_{n}-B_{n}\right) \cup A_{n}$ be called an $S_{n-s e t}(n=1,2, \cdots)$. 
Define an $S$-topology in $Z^{Y}$ by taking as a subbase the sets $(A, W)$ where $W$ is open in $Z$ and $A$ is an $R$-set or an $S_{n}$-set for some $n$. (The notation $(A, \mathbb{W})$, defined earlier, refers to the class of functions sending $A$ into $W$.) Designate this topology simply by " $S$ ".

We first show that $S$ is admissible.

Let $f$ and $y$ be given, as well as a neighborhood $W$ of $f(y)$. We consider two cases.

Case 1: $y=y_{0}$. There is a neighborhood $V_{n}$ on which $f$ has all its values in W. For each $x$ in $s_{n}$ obtain a neighborhood $B_{x}$ contained in $V_{n}-V_{n+1}^{-}$and avoiding the set $t_{n}$. Let $B_{n}$ be the union of these $B_{x}$. Then $\left(V_{n}-B_{n}, W\right)$ is a neighborhood of $f$, and for $g$ therein and $x$ in $V_{n+1}$ we surely have $g(x)$ in $\mathbb{W}$.

Case 2: $y=y_{n}$. We can find a neighborhood $A$ of $y$ which is an $R$-set and on which $f$ has values only in $W$. For $g$ in $(A, W)$ and $x$ in $A$ we have $g(x)$ in W. With the completion of this second case we have shown the admissibility.

Replacing each $S$ by a $T$, each $s$ by a $t$, and each $t$ by an $s$, regardless of subscripts, we obtain the definition and admissibility of another topology, $T$.

\section{(6.31) The meet $S \wedge T$ is not admissible.}

Suppose it were admissible. Let $\mathbb{W}$ be the complement of 1 in $Z$, and let $f_{0}$ be a function in $Z^{Y}$ such that $f_{0}\left(y_{0}\right)=0$ and $f_{0}(y)=1$ for some $y$ in $r_{1}$. Then there is a set $U$ open in both $S$ and $T$, and a $V_{p}$, such that:

(6.32) The relations $f \in U$ and $y \in V_{p}$ together imply $f(y) \in W$.

Continuing the proof of (6.3) we now deduce the following from (6.32):

(6.33) If $U$ contains an $f$ which assumes the value 1 on some point of $r_{m}$, then it contains an $f_{1}$ which assumes that value on some point of $r_{n}$ for some $n$ greater than $m$.

Proof. We may suppose that $f$ in $U$ assumes the value 1 on some point of $s_{m}$. Now $f$ has a neighborhood $U_{T}=\left(A_{1}, \cdots, A_{j} ; W_{1}, \cdots, W_{j}\right)$, where the latter expression denotes the intersection of $\left(A_{1}, W_{1}\right), \cdots,\left(A_{j}, W_{j}\right)$ in $T$. Some $W_{i}$ clearly excludes 1 , for otherwise the constant function 1 belongs to $U$, violating (6.32). Let $W_{1}, \cdots, W_{k}$ be those that exclude 1 , and suppose $A_{1}$ is a $T_{n}$-set with the lowest value of $n$. The closures of the finitely many $R$-sets figuring in $U_{T}$ clearly cannot cover $t_{n}$. Hence there is a point $y_{1}$ in $t_{n}$ with a neighborhood $V$ intersecting none of $A_{1}, \cdots, A_{j}$. We construct a continuous real-valued function $g$ with $g\left(y_{1}\right)=1-f\left(y_{1}\right)$, vanishing outside $V$, and having 0 and $1-f\left(y_{1}\right)$ as its bounds. Let $f_{1}=f+g$; this function has the property required by (6.33), but it remains to show that we have $n>m$. If we had $n \leq m$ then $s_{m}$ would be inside $A_{1}$. Now $f$ assumes the value 1 somewhere on $s_{m}$. Thus 1 belongs to $W_{1}$. This contradicts the earlier finding that $W_{1}$ does not contain 1 . Hence (6.33) is proved. 
To prove (6.3) we observe that from the $f_{0}$ and an iterated application of (6.33) we finally obtain an $f$ in $U$ which assumes the value 1 somewhere on $V_{p}$. This contradicts (6.32). Hence (6.3) is proved.

As in the case of (6.2), this result (6.3) can be extended automatically to the case in which $Z$ merely possesses a non-degenerate arc, and is a $T_{0}$-space. The "Sierpinski space" (see (4.6)) is an example. However, one can do a little better in the case of the Sierpinski space, as follows.

(6.4) THEOREM. Let $Y$ be a Hausdorff space in which each point has a countable basis, and let $Z$ be the Sierpinski space $\{0,1\}$ (see (4.6)). If the meet of every pair of S-admissible topologies in $Z^{Y}$ is admissible, then $Y$ is locally compact.

The reader can obtain the proof out of that of (6.3), observing these changes: (a) instead of having no limit points, the $r_{n}$ have no complete limit points (see Alexandroff and Urysohn [14, p.7]); (b) the $R$-sets may intersect each $r_{n}$ in a set of power less than that of $r_{n}$; (c) the value $j$ need not exceed 1 ; and (d) the sets $B_{n}$ may be taken as $t_{n}$. In fact, our method of investigating these matters was to consider first the case where $Z$ is the Sierpinski space.

The following observation is of interest. In (6.3) we saw that the meet $t$ of two admissible topologies may not have enough open sets to be itself admissible, unless $Y$ is locally compact. Although there are obviously topologies which are neither admissible nor proper, one might wonder whether any such are accessible through lattice operations from admissible topologies. In other words, if the $t$ above is not admissible is it necessarily proper? A consideration of the proof of (6.3) shows that the meet $t$ of $S$ and $T$ need not be proper: each set of the form $(A, \mathbb{W})$, where $A$ is an $R$-set, is open in $t$; but there is no reason why all $R$ sets should be compact, and hence (by (4.41)) why $t$ should be proper.

An observation which sometimes leads to the identification of the greatest proper topology is this: If the meet of two admissible topologies is proper, this meet is the greatest proper topology. An application of this to the reasoning of (6.3) yields a result which should be compared with the earlier example (5.3).

(6.5) The овем. When $Y$ is not locally compact, and $Z$ is the Sierpinski space $\{0,1\}$, then the k-topology may be the greatest proper topology.

Proof. Let $Y$ be the space of pairs of positive integers $(i, j)$ with an added point " $\infty$ ". Neighborhoods of $\infty$ shall be $V_{n}(n=1,2, \cdots)$ of points with $j \geq n$, plus $\infty$ itself. Other points are isolated. Define an $S$-topology as follows. Let $A_{n}$ be the set of points $(i, j)$ where $j \geq n+1$ when $i$ is odd and $j \geq n$ when $i$ is even, plus $\infty$ itself. Let $A_{\infty}$ be void. A set denoted by $B$ shall be any finite set. The $S$-topology shall be based on such $A$ or $B$ sets; call it $S$. The sets of the form $\left(A_{n} \cup B, 0\right)$, where $0 \in\{0,1\}$ is the open point, clearly form a basis. Interchange "odd" and "even" in the above and arrive at another topology $T$. Both these 
topologies are admissible, and hence $\geq k$, whence $S \wedge T \geq k$. Let $U$ be open in $S \wedge T$, and contain the function 0 . Select an $\left(A_{n} \cup B, 0\right)$ containing 0 and contained in $U$, the former being open in $S$. Then there must be an element $f$ in $U$ which has the value 1 at almost all points with $j<n$, and also at almost all points $(i, j)$ with $j=n$ and $i$ odd. Now select another neighborhood $\left(A_{p} \cup B_{1}, 0\right)$ of $f$ in $U$, the former being open in $T$. Then clearly $p>n$. There is then a $g$ in $U$ with value 1 at almost all points with $j<p$ and at almost all points $(i, j)$ with $j=p$ and $i$ even. By induction we arrive at a set $K$ of points, finitely many on each row, such that, if $h(i, j)=0$ for $j \geq N$ (any $N$ ) and for $(i, j) \in K$, then we have $h \in U$. Let $K_{1}=K \cup\{\infty\}$. What we have just said is that if $h \in\left(K_{1}, 0\right)$ then $h \in U$. Hence $U$ is open in $Z^{Y}(k)$. If in the first neighborhood $\left(A_{n} \cup B, 0\right)$ we have $n=\infty$, then we have already a $k$-open set containing 0 and contained in $U$. The argument is still simpler for any function which is not 0 identically. Thus we have $S \wedge T \leq k$, or $S \wedge T=k$.

It is probable that this example can be adapted to the case where $Z$ is the real interval $[0,1]$.

7. The space of closed sets. Let $S$ be the Sierpinski space of two points 0 and 1 , where $\{0\}$ is open and $\{1\}$ is not open. Consider $S^{Y}$ for any space $Y$. Let $f \in S^{Y}$, and let $F$ be the class of points on which $f(y)=1$. Then $F$ is evidently a closed set, and clearly every closed set can be obtained in this way. The notation of $S$ has been so chosen that the correspondence $f \leftrightarrow F$ preserves the lattice operations ( $S$ obviously is a lattice, and this introduces lattice ordering into $S^{Y}$ in an obvious way) and the Boolean ring operations (where we use intersection and symmetric difference $\left(F_{1} \cup F_{2}\right)-\left(\begin{array}{lll}F_{1} & \cap F_{2}\end{array}\right)$ in the class $F$ of closed sets $)$. We sum up this situation briefly as a theorem.

\section{(7.1) The OREM. SY and F are isomorphic.}

We shall henceforth prefer the symbol "ry", or "r子 $(Y)$ ", to " $S Y$ ", and shall write the elements as $F, F_{1}, \cdots$, using $F$ in a dual way when we write

(7.11) $y \in F$ if and only if $F(y)=1$.

Having decided to regard $\mathcal{F}$ as a space of continuous functions, we naturally investigate first the interpretation of any kind of convergence in 7 which does not require introduction of any topology in the function space.

(7.2) Тнеовем. Let $\left\{F_{\mu}\right\}$ be a directed set in $\mathcal{F}$. Then $F_{\mu}$ converges continuously (see circa 2.4) to $F$ if and only if $F \supset \lim \sup F_{\mu}$.

Before proceeding to the proof, we must explain what the lim sup of a directed set of sets $E_{\mu}$ is. Generalizing Hausdorff's definition (Alexandroff-Hopf [ 1 , p.111]) in an obvious way (see Choquet [6]) we say: 
(7.21) $y \in \lim \sup E_{\mu}$ if and only if for every $\mu$ and every neighborhood $V$ of $y$ there is a $\mu^{\prime} \geq \mu$ such that $E_{\mu \prime}$ intersects $V$.

We add the customary companion:

(7.22) $y \in \lim \inf E_{\mu}$ if and only if for every neighborhood $V$ of $y$ there is a $\mu$ such that for every $\mu^{\prime} \geq \mu, E_{\mu^{\prime}}$ intersects $V$.

Proof of 7.2. Let lim sup $F_{\mu}$ be denoted by $L$. Then $L(y)=0$ precisely if for some $\mu$ and some $V$ of $y$ and every $\mu^{\prime} \geq \mu$ we have $F_{\mu},(y)=0$. Now suppose that $F_{\mu}$ converge continuously to $F$ (the definition precedes (2.4)), and suppose $F(y)=0$. It follows at once that $L(y)=0$. Hence the continuous convergence implies $L \subset F$ (see (7.11)). Conversely, suppose $F \supset L$. To check continuous convergence we need only consider $y$ such that $F(y)=0$. Then $L(y)=0$. Reading the second sentence of this proof, we see that the condition of continuous convergence is satisfied.

Observe that continuous limits are not unique. Everything converges continuously to $Y$ itself, for example.

The condition that a topology for $\mathcal{F}$ should be admissible is easily deducible from (2.5) and (7.2).

(7.23) THEOREM. A topology $t$ for ${ }^{7}$ is admissible if and only if for every directed set of closed sets $\left\{F_{\mu}\right\}$ which converges to $F$ according to $t$ we have $F \supset \lim \sup F_{\mu}$.

We shall now consider the significance of proper topologies.

(7.3) ThEOREM. Let $X$ and $Y$ be spaces, and let $\Phi$ be a closed subset of $X \times Y$. For each $x$ let $F_{x}$ be the closed set of points $y$ for which $(x, y) \in \Phi$. Then a necessary and sufficient condition that a topology for $f$ be proper is that for every $\Phi$ the associated mapping

$$
f: X \rightarrow \mathcal{F} ; f(x)=F_{x},
$$

be continuous.

According to (6.25), (6.01), and (4.71), when $Y$ is a regular space we can obtain a topology $t$ with the properties of (7.23) and (7.3) if and only if $Y$ is locally compact, and that topology will be the $k$-topology. We wish to compare this topology with that introduced by Hausdorff into $\mathcal{F}$ when $Y$ is a compact metric space (see Alexandroff-Hopf [1]) and further generalized by Choquet [6, pp. 87-93]. Hausdorff's topology $H$ is surely not the same as the $k$-topology, for $S^{Y}(H)$ is a Hausdorff space whereas in $S^{Y}(k)$ the closed set $Y$ has, as its only neighborhood, F itself. Theorem VI (Alexandroff-Hopf $[1, \mathrm{p} .115]$ ) shows that convergence in $S Y(H)$ fulfills the condition of (7.23), so that $H$ is admissible, and thus $H>k$. As a matter of fact, the void set is omitted in Hausdorff's treatment; but a formal 
application of definitions shows that it would be isolated, as it is in the $k$-topology.

Since Hausdorff's topology $H$ makes f compact [1], there is no other Hausdorff topology $H^{\prime}$ lying between $k$ and $H$. This supports the conjecture that $H$ is the least admissible Hausdorff topology.

8. Topological products. The techniques of this paper enable us to give an answer to the following question: If $X$ is an arbitrary set and $Y$ a space, if $T$ is a topology in the set of all couples $(x, y), x \in X, y \in Y$, yielding a topological space $P$, when can a topology $t$ be introduced in $X$ so that $P$ is the topological product $X(t) \times Y$ ?

(8.1) THEOREM. With the notations as above, assume $T$ has the following properties:

(8.11) For each fixed $x_{0}$, the mapping $f_{x_{0}}(y)=\left(x_{0}, y\right)$ of $Y$ into $P$ is continuous;

(8.12) The mapping $g(x, y)=y$ of $P$ into $Y$ is continuous;

(8.13) Given any two points $\left(x_{0}, y_{0}\right),\left(x_{0}, y_{0}^{-}\right)$of $P$, and any neighborhood $V$ of $\left(x_{0}, y_{0}\right)$, there exists a neighborhood W of $y_{0}$ and a neighborhood $V^{\prime}$ of $\left(x_{0}, y_{0}^{-}\right)$ such that $\left(x, y^{-}\right) \in V^{\prime}$ and $y \in \mathbb{W}$ imply $(x, y) \in V$.

Then, if $P Y$ has a proper and admissible topology, there exists a topology $t$ in $X$ with $X(t) \times Y=P$.

Proof. Since by (8.11), for each $x, f_{x}(y) \in P^{Y}$, the map $F^{*}, F^{*}(x)(y)=f_{x}(y)$, is a one-to-one mapping of $X$ into $P Y$, and so in all that follows we shall consider $X \subset P^{Y}$. We now give $X$ the topology $t$ of a subset of the space $P Y$. Then we have $F^{*}: X(t) \rightarrow P^{Y}$; and, due to the admissibility, for the associated map we have $F: X(t) \times Y \rightarrow P$. It is evident that $F$ is the identity map.

On the basis of (8.13) we note that defining $h\left[y,\left(x, y^{-}\right)\right]=(x, y)$ we get $h: Y \times P \rightarrow P$; by the properness we find $h^{*}: P \rightarrow P^{Y}$, and it is easy to see that $h^{*}$ maps $P$ into $X \subset P^{Y}$, so that we have $h^{*}: P \rightarrow X$. Using (8.12), we also have $g: P \rightarrow Y$. Defining $H(x, y)=\left[h^{*}(x, y), g(x, y)\right]$, we thus have $H: P \rightarrow X(t) \times Y$, and $H$ is the identity map. Hence, from the above we find that $X(t) \times Y$ and $P$ are homeomorphic, and the theorem is proved.

Note that in case $Y$ is locally compact and regular, then from $(4.71)$ the $k$ topology in $P^{Y}$, for any space $P$, is admissible and proper.

(8.2) ТHe ORE arbitrary set. A necessary and sufficient condition that a topology $T$ in the set $X \times Y$ be a product topology with one factor the space $Y$, is that $T$ satisfy (8.11) through (8.13).

Proof. The necessity of (8.11) through (8.13) is immediate from elementary 
properties of topological products. The sufficiency of (8.11) through (8.13) stems from (4:71) and (8.1).

Another result on the behavior of topological products that is implied by our results will now be given. Let $Y$ be a fixed space, and $X$ a set carrying topologies $s$ and $t$. In the set of all pairs, $X \times Y$, let $S$ be the product topology of $Y \times X(s)$, $T$ the product topology of $Y \times X(t)$, and $R$ the product topology in $Y \times X(s \wedge t)$.

(8.3) ТнЕовЕM. In the set $X \times Y$, we always have $R \leq S \wedge T$. Furthermore:

(8.31) If $Y$ is locally compact and regular, then $R=S \wedge T$;

(8.32) If $Y$ is not locally compact, then in general $R \neq S \wedge T$.

Proof. If $W$ is open in $R$, and $(y, x) \in W$, then there is a set of the form $U \times V$, $U$ open in $Y, V$ open in $X(s)$ and in $X(t)$, with $(y, x) \in U \times V \subset \mathbb{W}$. This means that $U \times V$ is open in $S$ and in $T$, hence in $S \wedge T$. It follows that $W$ is open in $S \wedge T$, so that $R \leq S \wedge T$.

$A d$ (8.31). Let $P$ denote the set $X \times Y$ with topology $S \wedge T$. Since $Y$ is locally compact, $P^{Y}$ has, by (4.71), an admissible and proper topology. We first remark that for the identity map, we have $g: Y \times X(s) \rightarrow P$; due to properness, we have for the associated map $g^{*}: X(s) \rightarrow P^{Y}$. Using the same map, we also obtain $g^{*}: X(t) \rightarrow P^{Y}$, and so evidently we have $g^{*}: X(s \wedge t) \rightarrow P^{Y}$. By admissibility we find for the identity map $g: Y \times X(s \wedge t) \rightarrow P$. This shows that $S \wedge T \leq R$ and, with what we have already shown, this gives $S \wedge T=R$.

$A d$ (8.32). Let us take $Y$ to be the space of (6.4) and $X$ to be the set $Z^{Y}$ of (6.4). Let $s$ and $t$ be two admissible topologies whose meet is not admissible. We show that $R \neq S \wedge T$. First we note that the evaluation map $\omega$ of $Y \times Z^{Y}(s \wedge t)$ into $Z$ is not continuous in $R$ since $s \wedge t$ is not admissible. But $\omega$ is clearly continuous in $S \wedge T$, since it is continuous in both $S$ and $T$. This proves that $R \neq S \wedge T$, and also establishes the theorem.

In case both factors are allowed to change, the assertion (8.32) always holds, regardless of whether $Y$ is compact or not.

(8.4) THEOREM. Let $X$ be an arbitrary set, and let $s$ and $t$ be two topologies in $X$. If $S$ is the topology of $X(s) \times X(s)$, $T$ the topology of $X(t) \times X(t)$, and $R$ the topology of $X(s \wedge t) \times X(s \wedge t)$, then in general $R \neq S \wedge T$.

Proof. We take $X$ to be the countable set of (3.54) and $s$ and $t$ the two compact Hausd orff topologies mentioned there. We remark that it is trivial to prove that the diagonal $D=\{(x, y) ; x=y\}$ in a space $X(r) \times X(r)$ is closed if and only if $r$ is a Hausdorff topology To prove the theorem, we note that on the basis of this remark, $D$ is closed in $S$ and in $T$, hence in $S \wedge T$, but that $D$ is not closed in $R$ since $s \wedge t$ is not a Hausdorff topology. 


\section{REFERENCES}

1. P. Alexandroff and H. Hopf, Topologie I, Springer, Berlin, 1935.

2. R. Arens, A topology for spaces of transformations, Ann. of Math. 47(1946), 480-495.

3. - Note on convergence in topology, Math. Mag. 23 (1950), 229-234.

4. G. Birkhoff, Lattice theory, Amer. Math. Soc. Colloquium Publications, vol.25; American Mathematical Society, New York, 1948.

5. - The combination of topologies, Fund. Math. 26 (1936), 156-166.

6. G. Choquet, Convergences, Ann. Univ. Grenoble. Sect. Sci. Math. Phys. (N.S.) 23 (1948), 57-112. $429-432$.

7. R. H. Fox, On topologies for function spaces, Bull. Amer. Math. Soc. 51 (1945),

8. O. Frink, Topology in lattices, Trans. Amer. Math. Soc. 51 (1942), 569-582.

9. E. Hewitt, A problem of set-theoretic topology, Duke Math. J. 10 (1943), 309-333.

10. C. Kuratowski, Topologie I, Monografje Matematyczne, Warszawa-Lwow, 1933.

11. Sur la topologie des espaces fonctionels, Ann. Soc. Polon. Math. 20 (1947-48), 314-322.

12. Sur la notion de limite topologique d'ensembles, Ann. Soc. Polon. Math. 21 (1948-49), 219-225.

13. J. W. Tukey, Convergence and uniformity in topology, Princeton University Press, Princeton, 1940.

14. P. Alexandroff and P. Urysohn, Mémoire Sur Les Espaces Topologiques Compacts, Verh. Nederl. Akad. Wetensch. Afd. Natuurk. Sect. 1. 14 (1929), No. 1, 1-96.

\section{University of California, Los Angeles \\ University of Southern California}





\title{
EDITORS
}

\author{
HERBERT BUSEMANN \\ University of Southern California \\ Los Angeles 7, California
}

R. M. RoBINSON

University of California

Berkeley 4, California

E. F. BECKENBACH, Managing Editor

University of California

Los Angeles 24, California

ASSOCIATE EDITORS
R. P. DILWORTH
P. R. HALMOS
BQRGE JESSEN
J. J. STOKER
HERBERT FEDERER
HEINZ HOPF
PAUL LÉVY
E. G. STRAUS
MARSHALL HALL
R. D. JAMES
GEORGE PÓLYA
KÔSAKU YOSIDA

\section{SPONSORS}

UNIVERSITY OF BRITISH COLUMBIA

CALIFORNIA INSTITUTE OF TECHNOLOGY UNIVERSITY OF CALIFORNIA, BERKELEY

UNIVERSITY OF CALIFORNIA, DAVIS

UNIVERSITY OF CALIFORNIA, LOS ANGELES

UNIVERSITY OF CALIFORNIA, SANTA BARBARA

OREGON STATE COLLEGE

UNIVERSITY OF OREGON
UNIVERSITY OF SOUTHERN CALIFORNIA STANFORD UNIVERSITY

WASHINGTON STATE COLLEGE

UNIVERSITY OF WASHINGTON

AMERICAN MATHEMATICAL SOCIETY NATIONAL BUREAU OF STANDARDS, INSTITUTE FOR NUMERICAL ANALYSIS

\section{Vari-Type Composition by \\ Cecile Leonard \\ Ruth Stafford}

With the cooperation of

E. F. Beckenbach

E. G. Straus

Printed in the United States of America by

Edwards Brothers, Inc., Ann Arbor, Michigan

UNIVERSITY OF CALIFORNIA PRESS - BERKELEY AND LOS ANGELES COPYRIGHT 1951 BY PACIFIC JOURNAL OF MATHEMATICS 


\section{Pacific Journal of Mathematics}

\section{Vol. 1, No. $1 \quad$ November, 1951}

Ralph Palmer Agnew, Ratio tests for convergence of series............. 1

Richard Arens and James Dugundji, Topologies for function spaces....... 5

B. Arnold, Distributive lattices with a third operation defined ........... 33

R. Bing, Concerning hereditarily indecomposable continua ........... 43

David Dekker, Generalizations of hypergeodesics ............... 53

A. Dvoretzky, A. Wald and J. Wolfowitz, Relations among certain ranges of vector measures.................................... 59

Paul Erdős, F. Herzog and G. Pirani, Schlicht Taylor series whose convergence on the unit circle is uniform but not absolute .......... 75

Whilhelm Fischer, On Dedekind's function $\eta(\tau) \ldots \ldots \ldots \ldots \ldots \ldots . \ldots 3$

Werner Leutert, The heavy sphere supported by a concentrated force ...... 97

Ivan Niven and H. Zuckerman, On the definition of normal numbers ...... 103

L. Paige, Complete mappings of finite groups .................. 111

Otto Szász, On a Tauberian theorem for Abel summability ............. 117

Olga Taussky, Classes of matrices and quadratic fields ............. 127

F. Tricomi and A. Erdélyi, The asymptotic expansion of a ratio of gamma functions .................................... 133

Hassler Whitney, On totally differentiable and smooth functions ......... 143 